\title{
SEROLOGIC RESPONSE TO STREPTOCOCCAL HEMOLYSIN AND HYALURONIDASE IN STREPTOCOCCAL AND RHEUMATIC INFECTION
}

\author{
BY SUSANNA HARRIS AND T. N. HARRIS \\ (From The Children's Hospital of Philadelphia [Department of Pediatrics, School of Medicine, \\ University of Pennsylvania], Philadelphia, The Children's Seashore House, \\ Atlantic City, and the Philadelphia General Hospital, Philadelphia, Pa.)
}

(Received for publication August 24, 1949)

Although it has as yet not been possible to demonstrate the specific role of a causative organism in rheumatic fever, there has been considerable epidemiologic (1) and serologic (2) evidence of some relationship between the hemolytic streptococcus and this disease. Studies of both cellular and extracellular substances of the hemolytic streptococcus have with rare exceptions shown that groups of patients with acute rheumatic fever were indistinguishable, by serologic reactions, from streptococcal convalescents.

These studies have included those of reactions to cellular antigens, such as agglutination, precipitation with the $\mathrm{M}$ protein, and, more recently, complement fixation with somatic nucleoprotein fractions $(3,4)$; and, among the reactions to extracellular antigens, antistreptolysin, antifibrinolysin, and, most recently, antihyaluronidase.

A number of reports have shown that the sera of patients with acute rheumatic fever contained antibodies to streptococcal hyaluronidase in equal or greater concentration than those of patients convalescing from acute infection with the hemolytic streptococcus (5-10). This difference was manifest in both a higher geometric mean titer among the rheumatic fever patients and by a distribution of these titers which extended beyond the range of antihyaluronidase titers found after acute streptococcal infection. (The antihyaluronidase referred to here is the neutralizing antibody specific for hyaluronidase of streptococcal origin, and is stable at $56^{\circ} \mathrm{C}$. for 30 minutes. This point is made because in addition to the one or more thermolabile inhibitors of testicular hyaluronidase which have been described in human sera [11-14], thermolabile inhibitors of streptococcal hyaluronidase have been described which can be reactivated by complement $[15,16]$. The latter are distinct from the specific, thermostable inhibitor which has been measured in these studies.)
This difference between the antihyaluronidase titers in rheumatic and streptococcal infection has been discussed elsewhere (7). First, the factor of time was considered as a possible cause for this difference, since the streptococcal convalescents included in our series were studied three weeks after the onset of the disease, and it was felt that perhaps the longer contact with the streptococcus which some of the rheumatic patients may have had might have given rise to a greater production of this antibody. Other serologic circumstances which might give rise to such a difference were considered. Finally it was pointed out that only after thorough exploration of such possibilities could the question be raised as to whether the relatively higher antihyaluronidase titers in rheumatic fever might have any implication of some role of streptococcal hyaluronidase in the pathogenesis of this disease.

The current study was undertaken in an attempt to explore further the relationships of antihyaluronidase to scarlet fever and rheumatic fever, and where possible to compare it directly with the titers of antistreptolysin, in an effort to detect any possible relationship of antihyaluronidase to rheumatic fever and scarlet fever which was different from that of the more widely studied antistreptolysin.

For this purpose an effort was made this year to collect as many more sets of sera as possible from patients with scarlet fever at the onset of symptoms or shortly thereafter, three weeks later, and again six weeks later. Similarly, sera were collected from presumably normal subjects of the same age group, and also from patients with definite acute rheumatic fever in the same period of time. These were studied for comparison.

It was felt, in view of the widespread presence of the hemolytic streptococcus, and the variations which may be encountered from year to year in 
strains of the organism, that all sera in each of the clinical groups should be kept within groups arranged by years. From data previously reported (7), which contained material collected from 1942 to 1948 , those of 1947 and 1948 were taken, and to them were added the data of sera obtained in 1949.

\section{METHODS AND MATERIALS}

Serologic tests. Antihyaluronidase tests were done by both the mucin-clot-prevention (M.C.P.) and turbidimetric methods, as described elsewhere $(6,8)$. Since the results of these tests were directly parallel with each other the M.C.P. titers will be reported here, for comparison with previous data.

All sera were heated at $56^{\circ} \mathrm{C}$. for one-half hour prior to testing. In work done previously it had been found that the antibody measured against streptococcal hyaluronidase was heatstable (7), was to be found in the gamma globulin fraction of human sera (17), and was immunologically highly specific, showing no cross reaction with any of the hyaluronidases derived from bovine testis, pneumococci or clostridia (6).

The M.C.P. test is, under carefully controlled conditions, quite repeatable within one-half to one two-fold step of dilution of serum. All conditions of the test, including the effective concentrations of substrate and enzyme, were maintained as previously described. A number of procedures were followed in order to make the tests as comparable as possible. 1) The distribution of sera to be tested on a given day was always made in a similar pattern, one part being composed of sera from patients with acute rheumatic fever, one part sera from patients convalescent from scarlet fever, and the third part made up of sera from the normal population. This was done so that if on any given day the standard serum titers proved to be somewhat higher or lower than average no one clinical group would be affected more than the others. 2) Of 15 sera contained in each rack tested two sera were "standard" sera. They were tested daily for a given period prior to the long series of tests, as well as in the latter, and therefore any deviation in their titers beyond one-half two-fold step would serve to indicate some change had occurred in the sensitivity of that day's test. It was not found necessary to use correction factors. 3) In order to be certain of the validity of the comparisons to be made, large groups of sera tested in 1947 and 1948 were repeated with the set obtained in 1949, and the titers of these were checked against those previously obtained. It was found that on repeated titrations these sera showed titers identical with the original ones, or differing by half a two-fold step of dilution, in the case of 77 per cent of the rheumatic sera, and 81 per cent of the scarlet fever group. The remainder of each group showed titers differing by one two-fold step from the titration of the earlier years, or, in a very few cases, one and a half such steps.

Antistreptolysin tests were performed as reported elsewhere (4) by a slight modification of the original technic described by Todd. Serum dilutions were made in the same volume as those in the antihyaluronidase tests, so that direct comparisons could be made. Similar repetitions and other precautions were taken as in the case of the antihyaluronidase test.

Clinical material. The rheumatic patients studied were in the active stage of the disease. They were usually seen in the wards of The Children's Hospital of Philadelphia or of the Philadelphia General Hospital.

The patients were examined at least every week. These examinations included the following: symptomsanorexia, headache, precordial, abdominal, or arthritic pain, cough, dyspnea and epistaxis; physical signs-cardiac rate and rhythm, distance of apex beat from the mid-sternum, murmurs, with distance of transmission of each, and other adventitious sounds, palpable thrills and friction rubs, hepatic enlargement and edema, rashes, subcutaneous nodules and chorea. Laboratory examinations included the erythrocyte sedimentation rate, white blood cell count, and hemoglobin concentration.

Only those patients in whom the diagnosis of active rheumatic fever was beyond doubt were selected for serologic study. All of these children had active rheumatic carditis. Some of them were presumably in an initial episode of acute rheumatic carditis, whereas others were involved in a recurrence of the disease. There was no serologic difference, by the tests employed, between these clinical subdivisions. In approximately one-half of these episodes of rheumatic fever there was a history of a clear-cut upper respiratory infection about 10-20 days prior to the onset of rheumatic fever. However, these had in all cases occurred before hospitalization, and no bacteriologic data were available.

The patients with streptococcal infection were all hospitalized at the Philadelphia Hospital for Contagious Diseases for scarlet fever. Serum specimens were obtained one to four days after the onset of symptoms, at the end of the third week, and, in many instances, six to nine weeks after the onset of symptoms. None of these patients received chemotherapy. The disease was generally of mild or moderate severity although a number of these cases were more severe, with prolonged fever, albuminuria or toxic myocarditis. There was no correlation, however, between the severity of the disease and the titers in the two serologic tests reported here. The distribution of patients by age, color, and sex was quite similar in the streptococcal and rheumatic groups.

\section{RESULTS}

1. Geometric mean titers. Sera were obtained in 1949 from normal subjects of the age three to 12 years, from patients with scarlet fever three days and three weeks, respectively, after the onset of symptoms, and from patients with acute rheumatic fever. These were tested for their content of neutralizing antibodies to both streptococcal hyaluronidase and hemolysin, and the geometric 
TABLE I

Geometric mean titer

\begin{tabular}{|c|c|c|c|c|c|c|c|c|c|c|c|c|}
\hline \multirow{2}{*}{ Year } & \multicolumn{3}{|c|}{ Normal } & \multicolumn{3}{|c|}{ Scarlet fever acute } & \multicolumn{3}{|c|}{ Scarlet fever convalescent } & \multicolumn{3}{|c|}{ Acute rheumatic fever } \\
\hline & $\begin{array}{c}\text { Number } \\
\text { of } \\
\text { cases }\end{array}$ & $\mathbf{A H}^{*}$ & ASL* & $\begin{array}{c}\text { Number } \\
\text { of } \\
\text { cases }\end{array}$ & $\mathbf{A H}$ & ASL & $\begin{array}{c}\text { Number } \\
\text { of } \\
\text { cases }\end{array}$ & $\mathbf{A H}$ & ASL & $\begin{array}{c}\text { Number } \\
\text { of } \\
\text { cases }\end{array}$ & AH & ASL \\
\hline $\left.\begin{array}{l}1947 \\
1948 \\
1949\end{array}\right\}$ & $\begin{array}{l}185 \\
186\end{array}$ & $\begin{array}{l}36 \\
24\end{array}$ & $\begin{array}{l}30 \\
36\end{array}$ & $\begin{array}{r}104 \\
34 \\
64\end{array}$ & $\begin{array}{l}21 \\
13 \\
11\end{array}$ & $\begin{array}{l}39 \\
45 \\
17\end{array}$ & $\begin{array}{r}106 \\
36 \\
\cdot 150\end{array}$ & $\begin{array}{l}52 \\
56 \\
30\end{array}$ & $\begin{array}{r}120 \\
148 \\
98\end{array}$ & $\begin{array}{l}41 \\
33 \\
31\end{array}$ & $\begin{array}{l}482 \\
420 \\
366\end{array}$ & $\begin{array}{l}224 \\
240 \\
298\end{array}$ \\
\hline
\end{tabular}

* $\mathrm{AH}=$ antihyaluronidase.

$\mathrm{ASL}=$ antistreptolysin.

mean titer was calculated for each clinical group. These are shown in Table I, together with the geometric mean titers of analogous clinical groups observed during the years 1947 and 1948. Great care was taken to establish the validity of comparisons among titers obtained in the various years, in the case of each test.

It can be seen in Table I that the geometric mean antibody titer to streptococcal hyaluronidase and hemolysin found in acute rheumatic fever is higher for each year studied than that found in the group of patients convalescent from scarlet fever. This difference in mean titer between the patients with acute rheumatic fever and those convalescent from scarlet fever is always greater in the antihyaluronidase test than in the antistreptolysin. There are, however, fluctuations in the differences from year to year. In the year 1947 the ratio between the mean titers of the two clinical groups mentioned was 9.2 in the case of antihyaluronidase and 1.9 in the case of antistreptolysin; in 1948 these ratios were 7.5 and 1.6 respectively, and in 1949 were 13 and 3 respectively. In comparing the mean titers found in acute rheumatic fever by antihyaluronidase and antistreptolysin, it can be seen that the actual titers are quite parallel to each other, year by year. In reviewing the mean titers for the group of convalescents from scarlet fever in both tests, it is obvious that the mean titer found after scarlet fever was distinctly

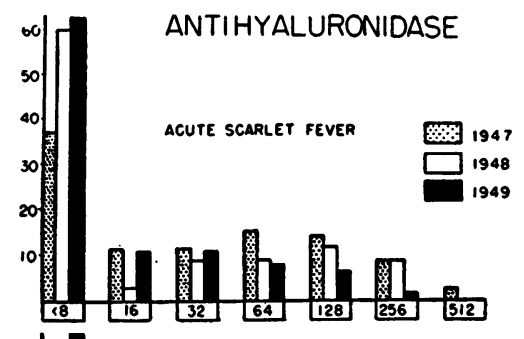

\section{ANTISTREPTOLYSIN}
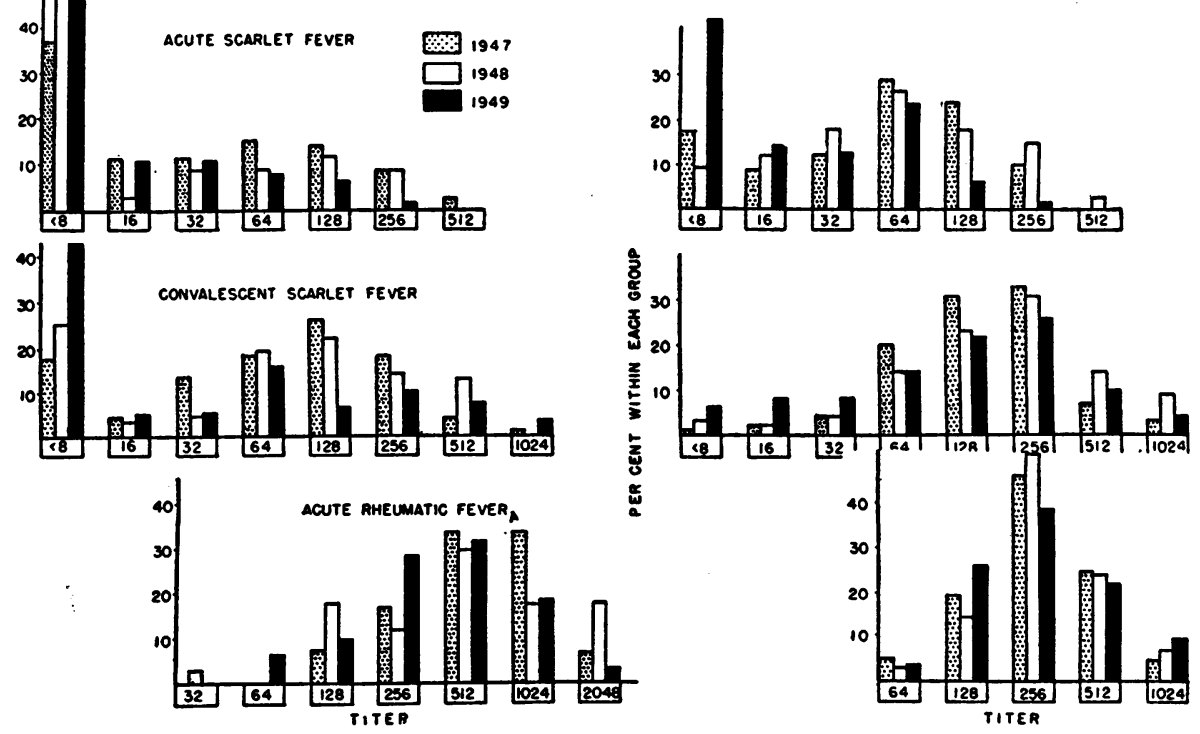

Fig. 1. Percentage-Frequency Distributions of Antistreptolysin and Antihyaluronidase Titrrs in Scarlet Fever and Active Rheumatic Fever, as Observed during Threx Successive Years 
lower in antihyaluronidase than in antistreptolysin. It was also noticed that the mean titers found in the acute phase of scarlet fever were often lower than those found in the normal population of the same age, both in the antihyaluronidase and antistreptolysin tests.

2. Percentage frequency distributions. The data obtained for three of the four clinical categories studied were broken down into the percentage frequency with which various antibody titers occurred in the antihyaluronidase and antistreptolysin tests. Figure 1 shows this distribution for each of the groups. Within each clinical group, the percentage of sera occurring with a given titer is indicated in the column where that titer is noted. The first block in each case represents 1947, the second, 1948, and the third, 1949.

In the distributions of titers of sera from patients during the acute phase of scarlet fever, it would seem that in general the range of titers encountered in both the antihyaluronidase and antistreptolysin tests was similar in the three years studied. In the years 1948 and 1949 there seemed to be a greater percentage of sera with less than measurable amounts of antibody in both tests (that is, titers below our arbitrary threshold of a $1: 8 \mathrm{di}$ lution of the serum to be tested). The distribution of titers in sera of patients convalescent from scarlet fever shows the change or rise in titer from the acute phase of the disease to the convalescent. In comparing the antihyaluronidase titers of the different years, a tendency was noted toward greater breadth in the range of titers encountered in 1948 and 1949. These years also produced many more sera with less than measurable amounts of antibody. The greater breadth of distribution, coupled with increased number of low-titered sera, leaves the middle group of titers with smaller percentages of the total. These differences are not apparent among the antistreptolysin titers found in the respective years.

Study of the distributions of titers of both antibodies among rheumatic patients shows a fairly constant pattern for the antistreptolysin test and a somewhat less constant one for the antihyaluronidase test. In 1949 there appears to be a somewhat greater clustering about the middle zone of titers in the antihyaluronidase test, with fewer sera at the very high titers listed. It is of interest that in both tests studied the sera of pa-
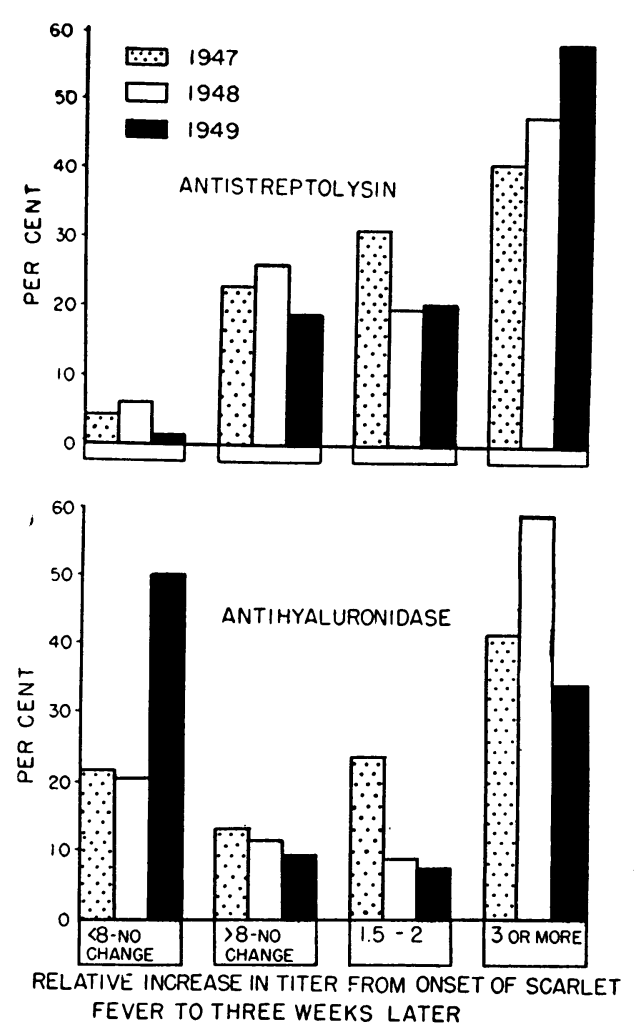

Fig. 2. The Changes in Antistreptolysin and Antihyaluronidase Titers Between the First and Third Weeks of Scarlet Fever as Observed in 1947, 1948 AND 1949

The increases are expressed as ratios of titers (convalescent: acute). Only increases of three or more are considered significant rises.

tients with acute rheumatic fever rarely contained low amounts of either antibody. Thus, in these sera none were encountered with less than measurable amounts of the antihyaluronidase or antistreptolysin, which is not true of convalescents from scarlet fever.

3. Study of the changes in titer from acute stage to convalescence in scarlet fever. The finding of such a large percentage of sera from patients convalescent from scarlet fever with antihyaluronidase titers of less than 8 warranted further study of such sera, in comparison with those obtained from the same patients during the acute phase of the disease. Pairs of sera collected in previous years were studied, as well as 64 pairs obtained during the wave of scarlet fever in the later winter and early spring of 1949 . (The diagnosis of scarlet fever was made at the Philadelphia Hospital for 
Contagious Diseases. Such diagnoses have in former years been corroborated by their bacteriologic studies in 95 per cent of their cases.) The pairs of sera taken in the acute and convalescent stages of the infection were always tested together and usually in succession in a given test, in order to obtain as valid a comparison as possible.

It was found that for the years studied most of the patients showed a rise in titer from the first to the third week after onset of symptoms. This change varied, however, from year to year and in the two tests. In Figure 2 are summarized the changes in titer occurring in the three years studied by the two tests. The pairs of sera in which both acute and convalescent patients showed titers below our threshold of measurement, thus precluding a comparison between them, are shown in the first set of columns. Sets of sera having measurable titers in the first specimen and no change in titer in the second specimen are indicated in the second series of columns; those showing an increase in titer to one and one-half or two times the titer of the first serum are in the next set of columns and finally those pairs of sera showing a three-fold or greater rise in titer. The last group included increases in titer in both tests up to 128 times the titer of the first serum. Although those sets of sera showing one and onehalf to two-fold rise in titer cannot be said to show a significant degree of difference, they are listed apart from the group actually showing no increase because these small differences were always in the direction of increasing titer. It was felt that random errors would have resulted in a slightly decreased titer for some pairs of sera and an increase for others.

Figure 2 shows that in all the years studied there were greater percentages of patients with scarlet

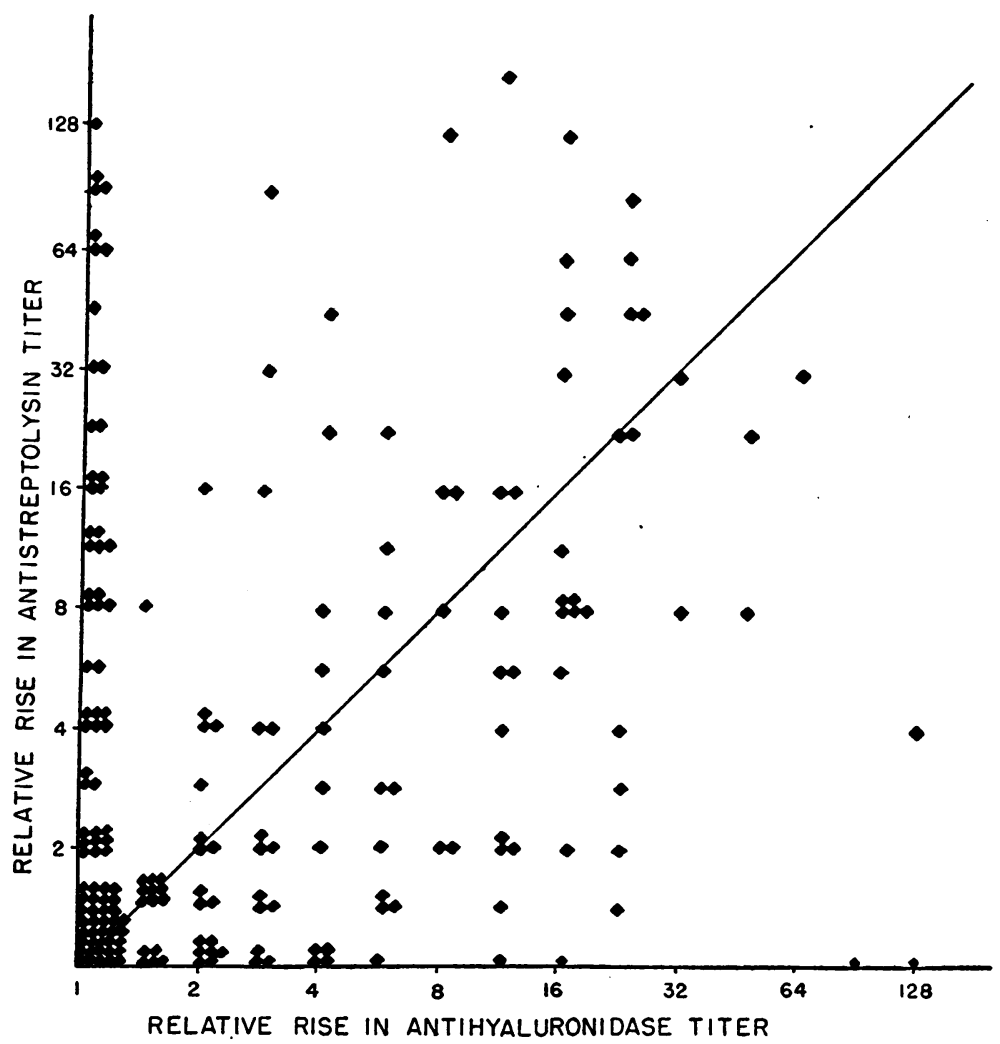

Fig. 3. The Rise in Antihyaluronidase Titer Compared with That in Antistreptolysin in Individual Patients Examined at the Onset of Scarlet Fever and Three Weexs Thereafter

Each point represents the pair of sera derived from one patient, the abscissa of the point corresponding to the ratio of antihyaluronidase titers and the ordinate to that of the antistreptolysin titers (convalescent: acute). 
fever who showed no change in the antihyaluronidase test than was true of the antistreptolysin test. In addition, of those sets of sera showing no change in titer during convalescence, it can be seen that the greatest number in the antihyaluronidase test occurred in the group having less than measurable amounts of antibody. In the case of antistreptolysin, however, most of those sets showing no change had measurable amounts of antibody in the serum obtained during the acute phase. Reviewing this from 1947 through 1949, it can be seen that relatively the greatest percentage of sera showing less than 8 titer against hyaluronidase in both specimens of serum occurred in 1949. This fact reflected itself in a lower geometric mean titer for the scarlet fever convalescent sera collected in 1949, as was seen in Table I.

In order to correlate the response of the individual patient in both the antihyaluronidase and antistreptolysin tests, the changes occurring from the acute phase to convalescence were plotted. Each point in Figure 3 represents one patient, and shows the rise in antistreptolysin as ordinate plotted against the rise in antihyaluronidase as abscissa. The points along the diagonal line indicate the sera which showed the same relative increase in titer in both tests. The three years covered are all plotted together since there was little apparent difference from year to year. The points indicating no change in titer in both tests include the group showing titers of less than 8 as well as measurable titers at the onset of the disease.

Judging by the wide scatter of points, there does not seem to be any relation between the antigenic response of an individual during scarlet fever to streptococcal hyaluronidase and hemolysin. Few dots fall on the diagonal line indicating similar change in the two tests. On the whole, there would appear to be more instances where a rise in antistreptolysin titer occurs as a result of the infection without change in the antihyaluronidase titer, than the reverse. The latter is seen to occur, but in fewer cases.

4. Study of the three to six week period following the onset of scarlet fever. In our observation of patients with scarlet fever, it had always been felt desirable to follow the serologic response beyond the third week. Since hospitalization of children for this disease lasts only through the third week in this community, it is somewhat diffi- cult to obtain further specimens of serum. Because of the questions raised by the antihyaluronidase titrations of recent years, an especial effort was made this year to obtain sera six weeks after onset of symptoms in some of these children. Although there were such sera available from previous years, our report includes only those collected in 1949 . The numbers are relatively small but may shed some light on the factor of time in the serologic response to these antigens.

A total of 55 cases were followed through the sixth week after the onset of symptoms of scarlet fever. Comparison was made of the titers found at three weeks and at six weeks. Here again sera were tested in pairs in the same racks to obtain the most valid comparison. The changes occurring from three weeks to six weeks were grouped as follows: those sera showing a decrease in titer of three- to eight-fold, those showing a decrease of one and one-half to two-fold, those having titers of less than 8 in both the third and sixth weeks, those sera with measurable titers in the third week and no further change thereafter, those sera showing a rise from the third to the sixth week of one and one-half to two-fold, and finally those showing a three-fold rise or more during that interval. The last category included one instance of an eightfold rise in antihyaluronidase, but the greatest increase in antistreptolysin was six-fold. The percentages are given in Figure 4. The findings

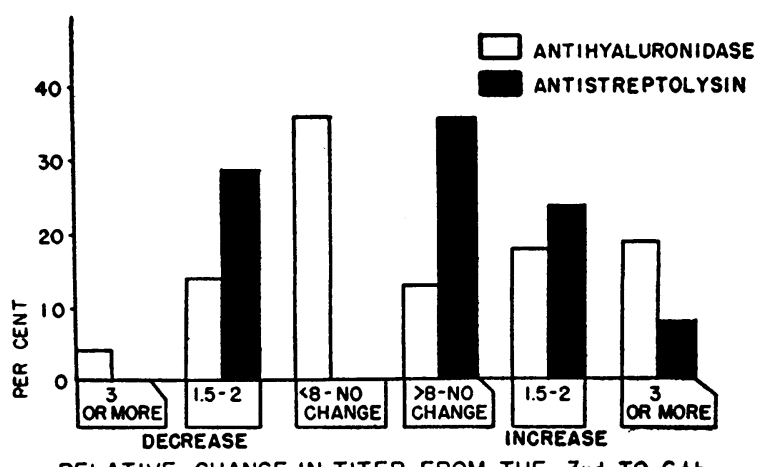

RELATIVE CHANGE IN TITER FROM THE 3rd TO 6 th WEEK AFTER ONSET OF SCARLET FEVER

Fig. 4. The Relative Change in Antistreptolysin and Antihyaluronidase Titers Between the Third to the Sixth Week Following the Onset of Scarlet Fever, as Observed in 1949

Only increases of three or more are considered significant rises. 
for antihyaluronidase are listed in a column beside those for antistreptolysin.

At the sixth week 19 per cent of the cases showed a definite rise in antihyaluronidase, in comparison with the third week following the onset, as against 8 per cent in antistreptolysin. In the antihyaluronidase test 18 per cent showed some rise, but since they were all only one and a half to two-fold rises they are not considered significant. Twenty-three per cent showed a similar rise in the antistreptolysin test. Sixty-nine per cent of the sera tested in the antistreptolysin test showed either no further change after the third week or a definite decrease in titer. It is somewhat difficult to evaluate this in the case of the antihyaluronidase test because of the frequency of low-titered sera. Twenty-seven per cent of the sera measured in the antihyaluronidase test showed no change or some decrease in titer. In addition, 36 per cent having titers of less than 8 at three weeks showed no change in titer at the sixth week. If the latter two groups are added together, they would yield a total of 63 per cent showing no change or some decrease in titer.

It would then seem that most sera have reached their highest titer of both antihyaluronidase and antistreptolysin by the third week, and show much less change from the third to the sixth week. This seems to be somewhat more true of antistreptolysin than of antihyaluronidase for the sera collected in 1949.

\section{DISCUSSION}

A number of points of interest arise from consideration of the data presented above. First, from the point of view of the epidemiology and serology of streptococcal disease, it is of some interest that the pattern of serologic response in the community is fairly similar, to two streptococcal antigens at least, during successive years. (In one of these years, 1949, there was an unusually extensive wave of scarlet fever in Philadelphia.) It is worth noting that this is the case in spite of the complete lack of correlation of antihyaluronidase to antistreptolysin titer in the individual case.

The distributions of titers of the two antibodies may reflect differences in the production of the respective antigens by strains of the hemolytic streptococcus prevalent in the community. Although antistreptolysin titers below our arbitrary threshold of 8 were to be found in the normal and even in the convalescent streptococcal group, these gave the appearance of being a part of a continuous distribution, allowing for the fact that several successive two-fold dilutions of serum are included in this group. In the case of antihyaluronidase, however, the normal and even the post-streptococcal groups presented a rather discontinuous distribution, with many sera below measurable titer, then an apparent gap, followed by a fairly normal distribution of titers of 32 and above. This would seem to indicate that the strains of hemolytic streptococcus present in the community are, in general, relatively good producers of hemolysin, at least in terms of the amounts necessary to stimulate antibody production, so that the casual contacts with the organism in the normal population, and the brief contact of acute streptococcal infection would suffice to induce the production of measurable concentration of serum antibody in the large majority of subjects. In the case of hyaluronidase, however, it might well be that this enzyme is produced by the prevalent streptococci in a much lower range of amounts, so that a large group of subjects produce extremely small amounts of antihyaluronidase. In contrast to this, the distribution of antihyaluronidase titers in rheumatic fever, which includes no very low titers, might imply that a more prolonged or greater degree of contact with the hemolytic streptococcus characterizes those streptococcal infections which are followed by rheumatic infection. An alternative possibility is that rheumatic infections might tend to follow streptococcal infections caused by strains of the hemolytic streptococcus which produce more hyaluronidase. This last possibility is considered less likely in view of the report by Friou (9) of rheumatic reactivations associated with strains of streptococci poor in hyaluronidase production. Unfortunately in our work cultural studies were not feasible, and we know nothing of the hyaluronidase production by the hemolytic streptococcus involved.

The data are also of interest with respect to both a theoretical and practical consideration of the relationship of streptococcal antihyaluronidase to rheumatic fever. In theoretical terms, the question has arisen as to whether the higher mean antihyaluronidase titer in rheumatic fever might imply 
some role of streptococcal hyaluronidase in the pathogenesis of this disease. In our discussion of this question (7), it was pointed out that this elevated mean titer might well merely involve a serologic circumstance, based on a difference in time of maximal serologic response to hyaluronidase, or rate of liberation of this antigen, and that only if such consideration could be ruled out could the suggestion be made that the relatively higher antihyaluronidase titer in rheumatic fever than following streptococcal infection, in comparison with antistreptolysin titers, might have some etiologic implication. The data presented here offer some evidence consistent with the former hypothesis, in that the serologic response to hyaluronidase in an acute streptococcal infection, scarlet fever, is smaller than that to streptococcal hemolysin, as indicated by the differences in individual sera drawn in the acute and convalescent stages of the disease. This raises the possibility that the difference between the titers of the two antibodies in the two diseases may reflect in part a relatively lower antihyaluronidase titer in scarlet fever rather than some specific elevation of antihyaluronidase titer in rheumatic fever. Another factor which may be responsible for a part of this difference is the increase in antihyaluronidase titers in some of the streptococcal convalescents after the third week following the onset of such infections. This might favor the occurrence of higher antihyaluronidase titers in those patients with acute rheumatic fever who are seen for the first time more than three weeks after a foregoing streptococcal infection. This factor, if it was indeed significant, could not have had more than a limited effect, since only a minority of the patients with scarlet fever showed a rise in titer of antihyaluronidase after the third week (a smaller number showed a rise in antistreptolysin as well, during this time) and because a substantial number of our patients with acute rheumatic fever were seen two to three weeks after clearly recognizable upper respiratory infections.

In this connection it may be relevant to refer to data currently in press on antihyaluronidase titers in acute glomerulonephritis. In this study, it has been found that whereas some low titers of streptococcal antihyaluronidase were found in cases of acute glomerulonephritis, the upper end of the frequency distribution of these titers in this disease was fairly similar to that found in acute rheumatic fever during the same year (18). The observation of such a distribution of these titers in another disease, but one of similar streptococcal epidemiology, would be consistent with the concept that the relatively higher antihyaluronidase titers in rheumatic fever are not specifically related to the pathogenesis of the disease, unless rheumatic fever and glomerulonephritis are related in their pathogenesis more closely than is realized at present.

In practical terms, these data on the year-byyear variations of streptococcal antihyaluronidase in streptococcal and rheumatic disease serve to add a further limitation to the diagnostic applications of the antihyaluronidase test. One of the applications of this test is the detection of antihyaluronidase titers so high as to be above the range of streptococcal convalescents, and which therefore point to a diagnosis of rheumatic fever in patients who may be clinically suspected of having that disease. The usefulness of this application depends, naturally, on the extent to which the distribution of these titers in this disease extends beyond that found after streptococcal disease. Our data show that despite general year-to-year similarities in the frequency distributions, annual variations occur. While these variations are small, they may have considerable effect on the degree of usefulness of the test in any one year. Thus in 1949 the relative difference in mean antihyaluronidase titer between the two diseases in Philadelphia was slightly higher than in 1947 or 1948 . Nevertheless, an important limiting factor to the diagnostic application of this test during that year was the circumstance that the frequency distribution curve of antihyaluronidase titers in acute rheumatic fever was of a narrower form, both high and low extremes being less prevalent than in earlier years, while the analogous distribution in scarlet fever was, on the contrary, wider than usual.

It will be seen, then, that the antihyaluronidase test, as applied to acute rheumatic fever, is subject to considerable limitation and requires careful interpretation. The optimal evaluation of the titer calls not only for clinical information concerning the patient but, for all but the extremely high titers, some knowledge of the distribution of these titers in the streptococcal convalescents of the community during the current year. It is ob- 
vious that the streptococcal antihyaluronidase test cannot be considered a test to be included in the armamentarium of hospital laboratories in general, but should probably be carried out, for the best of its limited applications, in conjunction with studies of rheumatic fever by laboratories engaged in such investigations, and in combination with serologic tests involving other streptococcal antigens, such as the hemolysin.

\section{SUM MARY}

Antistreptolysin and streptococcal antihyaluronidase titers have been determined in the sera of patients with acute rheumatic fever and streptococcal infection collected in the winter and spring of 1948-1949. The geometric mean titers of the different clinical groups and the frequency distribution of such titers analyzed for three successive years show general similarities from year to year, with minor differences within the distributions for each disease.

The geometric mean titers of the sera from patients with rheumatic fever were higher than those found after scarlet fever in both the antihyaluronidase and antistreptolysin tests. This difference varied somewhat from year to year in each test.

Study of pairs of sera obtained from patients at the onset of symptoms of scarlet fever and three weeks later showed a wide range of variation in the degree of serologic response to each antigen. There was no correlation between the increase of antistreptolysin and of antihyaluronidase titers in individual patients convalescent from scarlet fever. On comparing the sets of data obtained by each test it was found that a greater number of patients showed no increase in the titer of antihyaluronidase than of antistreptolysin. This was also reflected in the fact that many convalescents from scarlet fever had very low antihyaluronidase titers, whereas all of these titers in active rheumatic fever were well within the measurable range. This difference was much less marked in the case of antistreptolysin. These differences in serologic response are discussed in terms of the production of the antigens by prevalent strains, degree of contact between organism and host, and possible relationship between hyaluronidase and rheumatic fever.
Annual variation in the mean antihyaluronidase titers in acute streptococcal infection and active rheumatic fever, and especially in the breadth of distribution of this titer in the two diseases, is shown to be a possible source of increased limitation in the diagnostic application of this test.

\section{ACKNOWLEDGMENT}

We are indebted to Dr. A. C. LaBoccetta for permission to obtain specimens of serum of patients with scarlet fever in the Philadelphia Hospital for Contagious Diseases.

\section{BIBLIOGRAPHY}

1. Paul, J. R., The Epidemiology of Rheumatic Fever and Some of Its Public Health Aspects. Metropolitan Life Insurance Company, New York, 1943.

2. Harris, T. N., Studies in the relation of the hemolytic streptococcus to rheumatic fever. I. Review of serologic literature. Am. J. Dis. Child., 1948, 76, 411.

3. Harris, T. N., Ibid. II. Fractionation of the hemolytic streptococcus by high-speed centrifugation. J. Exper. Med., 1948, 87, 41.

4. Harris, T. N., Ibid. III. Complement fixation versus streptococcal nucleoproteins in sera of patients with rheumatic fever and others. J. Exper. Med., 1948, 87, 57.

5. Quinn, R. W., Antihyaluronidase studies of sera from patients with rheumatic fever, streptococcal infections and miscellaneous non streptococcal diseases. J. Clin. Invest., 1948, 27, 471.

6. Harris, T. N., and Harris, S., Studies in the relation of the hemolytic streptococcus to rheumatic fever. V. Streptococcal antihyaluronidase (mucinclot-prevention) titers in the sera of patients with rheumatic fever, streptococcal infection and others. Am. J. M. Sc., 1949, 217, 174.

7. Harris, T. N., Harris, S., and Nagle, R. L., Studies in the relation of the hemolytic streptococcus to rheumatic fever. VI. Comparison of streptococcal antihyaluronidase with antibodies to other streptococcal antigens in the serum of patients with rheumatic fever and acute streptococcal infection: mucin clot prevention test. Pediatrics, 1949, 3, 482.

8. Harris, T. N., and Harris, S., Turbidimetric measurement of streptococcal antihyaluronidase in the sera of patients with streptococcal infection and rheumatic fever. J. Immunol., 1949, 63, 249.

9. Friou, G. J., and Wenner, H. A., On the occurrence in human serum of an inhibitory substance to hyaluronidase produced by a strain of $H$. streptococcus. J. Infect. Dis., 1947, 80, 185.

10. Friou, G. J., Further observations of an inhibitor in human serums of the hyaluronidase produced by a strain of hemolytic streptococcus. J. Infect. Dis., 1949, 84, 240. 
11. Haas, E., On the mechanism of invasion. I. Antinvasin I, an enzyme in plasma. J. Biol. Chem., 1946, 163, 63.

12. Dorfman, A., Ott, M. L., and Whitney, R., The hyaluronidase inhibitor of human blood. J. Biol. Chem., 1948, 174, 621.

13. Glick, D., and Moore, D. H., Hyaluronidase inhibitor in electrophoretically separated fractions of human serum. Arch. Biochem., 1948, 19, 173.

14. Hadidian, Z., and Pirie, N. W., The effects of serum and of hyaluronic acid derivatives on the action of hyaluronidase. Biochem. J., 1948, 42, 266.

15. Thompson, R. T., and Moses, F. E., Thermostable in- hibition of bacterial hyaluronidases by the serum of normal human beings. Science, 1949, 110, 70.

16. Marcus, S., and Fulton, J. K., Relation of complement to thermolabile hyaluronidase inhibitory of serum. Federation Proc., 1949, 8, 407.

17. Moore, D. H., and Harris, T. N., Occurrence of hyaluronidase inhibitors in fractions of electrophoretically separated serum. J. Biol. Chem., 1949, 179, 377.

18. Harris, T. N., Harris, S., Dannenberg, A. M., Jr., and Hollander, J. L., Streptococcal antihyaluronidase titers in the sera of patients with rheumatoid arthritis and glomerulonephritis. Ann. Int. Med., in press. 